\title{
Management of pigmented skin lesions during pregnancy
}

Erica B Friedman, Richard A Scolyer, John F Thompson

\section{Background \\ As a result of the rapidly changing hormonal milieu, changing or newly discovered pigmented skin lesions during pregnancy can be diagnostically challenging. It is important for GPs to be aware of the effect of gestational changes on pigment production and features that should raise concern.}

\section{Objective}

The aim of this article is to provide an understanding of common changes that may occur in pigmented lesions during pregnancy, features that are of concern and the management of suspected melanoma in pregnant women.

\section{Discussion}

In pregnant women, changing naevi should be evaluated using conventional ABCDE melanoma diagnostic criteria, and suspicious lesions should not be attributed solely to a change in the hormonal milieu. In this population, diagnosed melanoma is probably best treated at a specialist centre.
PREGNANCY is a period of growth and change in many of a woman's organs, including her body's largest organ: the skin. With hormone-induced changes occurring in pigment production, evaluation of pigmented lesions in pregnancy can be difficult. The aim of this article is to describe common changes that can occur in pregnancy in pre-existing pigmented skin lesions, the assessment of new lesions that may appear during pregnancy and features of pregnancy-associated melanoma to aid the general practitioner (GP) in diagnosis and management of these lesions.

\section{Pigmented lesions in pregnancy}

Physiological changes in melanin pigment production due to oestrogen stimulation and higher-than-normal levels of melanocyte-stimulating hormone during pregnancy are responsible for common pregnancy-induced pigmentary phenomena including melasma on the face (Figure 1) and linea nigra on the abdomen (Figure 2). Historically, it was suggested that these pregnancy-related hormonal changes might accelerate growth or cause changes in benign melanocytic naevi. Some studies have reported an increase in size and/or darkening of some naevi during pregnancy, as also can occur with the use of oral contraceptives. An increase in size of naevi during pregnancy can be directly attributed to skin stretching and increased vascularity, most commonly in lesions on the abdomen and breasts. On

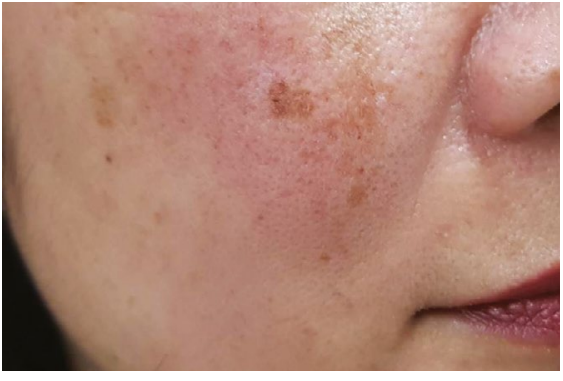

Figure 1. Melasma: Dark, irregular, welldemarcated patches of hyperpigmentation, often developing in pregnancy as a result of hormonal stimulation of melanocytes

dermoscopic examination, pigmented lesions may show changes in the pigment network or vascular structure that often return to normal within 12 months of childbirth (Box 1). ${ }^{1,2}$ While enlargement and darkening of melanocytic naevi occur in more than $10 \%$ of women during pregnancy, primarily in the first trimester, ${ }^{3}$ there is no evidence of an increased rate of malignant transformation. ${ }^{4}$ However, naevi excised from pregnant patients can display a mild degree of histological atypia or mitotic activity (Figure 3), supporting a theory of cellular activation during pregnancy. ${ }^{4}$ Nevertheless, the presence of prominent architectural disorder or cytological atypia in a naevus that has been excised from a pregnant woman should not be dismissed as a 'pregnancy effect'. Occasionally, pathological examination of otherwise typical benign naevi reveals 
the presence of frequent dermal mitoses, which often raises concern that the lesion may in fact be a subtle naevoid melanoma. Unless the history of concurrent or recent history of pregnancy is known, naevi with mitoses are at risk of being overdiagnosed as melanoma. To mitigate this risk, it is critical that the pathology request form should state clearly that the patient is pregnant or has given birth in the past 3-6 months. When genuine doubt exists about whether a melanocytic tumour is benign or malignant despite careful clinicopathological correlation and histological evaluation, molecular testing of the lesion may be warranted. This will provide additional evidence of the lesion's malignant potential, but even this is not always definitive.

One study showed that in women with dysplastic naevus syndrome (Figure 4), clinical change in naevi was 3.9 times greater in pregnancy than in the non-pregnant state. ${ }^{5}$ However, changes that would raise concern about the possibility of melanoma in a non-pregnant woman, or the appearance of new, clinically-concerning

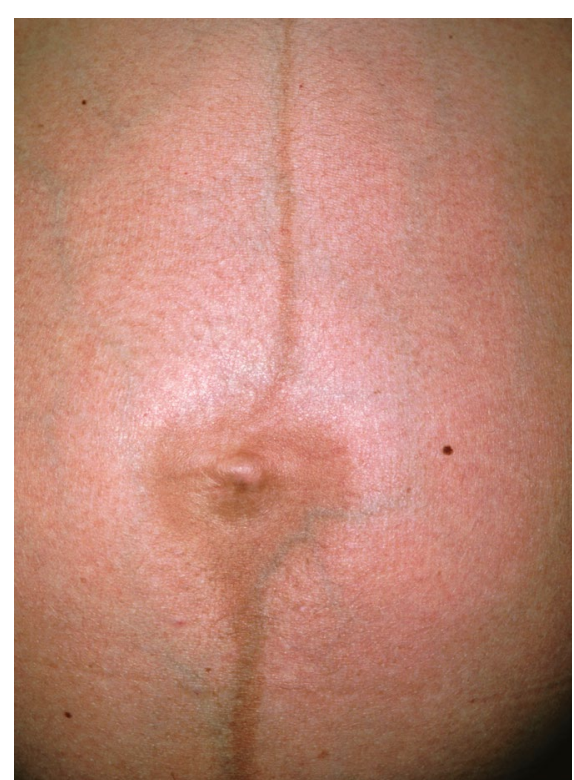

Figure 2. Linea nigra: A line of hyperpigmentation that often develops during pregnancy as a result of increased melanocyte-stimulating hormone

Figure 2 courtesy of Science Photo Library pigmented lesions should not be attributed to gestational changes and should instead prompt immediate biopsy.

\section{Melanoma in pregnancy \\ Epidemiology}

Pregnancy-associated melanoma (PAM) has been defined as a diagnosis of melanoma made during pregnancy or in the first 12 months postpartum. Melanoma is the most common malignancy in pregnancy, accounting for one-third of pregnancy-associated malignancies in Australia. ${ }^{6}$ Interestingly, the majority of these melanomas are diagnosed postpartum, ${ }^{6}$ highlighting the challenges associated with diagnosing cancer in pregnancy. The reported incidence of PAM in Australia increased from 37.1 to 51.84 per 100,000 from 1994 to 2008 , mostly attributed to increasing maternal age. ${ }^{7}$

\section{Presentation and diagnosis}

PAM presents a clinical challenge. Diagnostic delays may account for

\section{Box 1. Pregnancy-related dermoscopic changes in pigmented lesions}

\footnotetext{
- Progressive shading at borders

- Reduction in number and size of globules

- Increase in dotted vessels

- Increase in total dermoscopic score
}
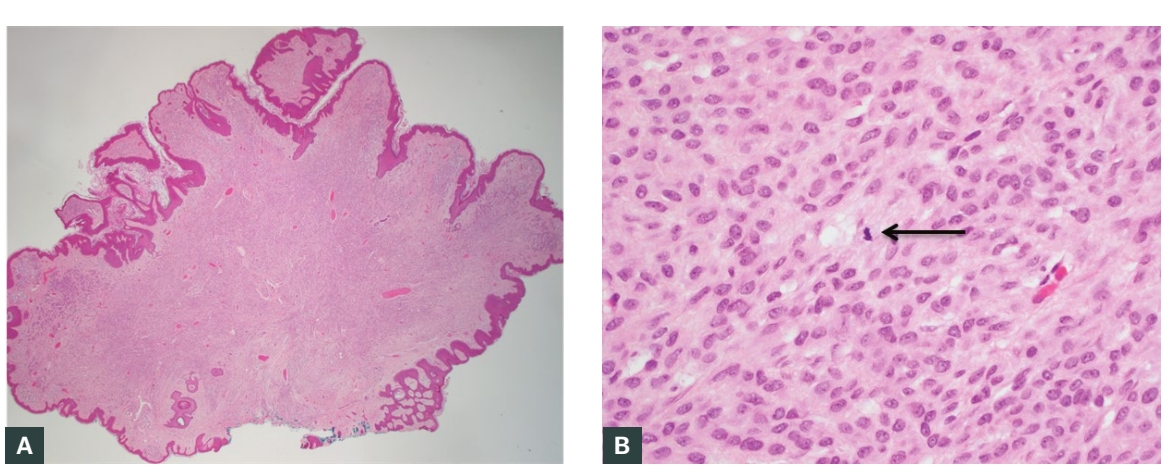

Figure 3. Histological images of a naevus excised from a pregnant woman and stained with haemotoxylin and eosin; benign naevi excised from pregnant patients can show a mild degree of atypia and/or mitotic activity, as indicated by the black arrow in Panel B

A. Low power; B. High power increased thickness of PAMs when compared with non-pregnant matched controls. ${ }^{8}$ All changing naevi in pregnancy should be monitored carefully using conventional ABCDE diagnostic criteria for melanoma, that is, if a naevus is noted to have Asymmetry, Border irregularity, Colour variegation, Diameter $>6 \mathrm{~mm}$ and/or Elevation (or Evolution in size, shape or colour). As for non-pregnant patients, if there is any concern, an excisional biopsy should be performed to allow thorough histological evaluation.

\section{Management}

There are no current national or international guidelines for the management of PAM, although consensus guidelines have been developed by Cancer Council Australia. ${ }^{9}$ The current standard is to treat melanocytic lesions in pregnant women as in non-pregnant women. Surgery remains first-line treatment; however, if possible, this should be delayed until the second trimester to avoid the small increased risk of miscarriage or fetal damage from anaesthetic agents in the first trimester. Pregnancy is not a contraindication to sentinel node biopsy, and the indications for sentinel node biopsy are the same as for non-pregnant patients. While lymphoscintigraphy with technetium-99m is safe in pregnancy, ${ }^{10}$ intraoperative blue dye mapping should be avoided because of the possible risk of teratogenicity and a small risk of anaphylaxis. 
Surgery is also the mainstay of treatment for patients with more advanced disease diagnosed in pregnancy, including therapeutic regional lymphadenectomy for palpable lymphadenopathy, and surgical resection for appropriately selected patients with isolated metastases. Though effective systemic therapy in both adjuvant and metastatic settings is now available, there is no evidence regarding the safety of either immunotherapy or targeted agents in pregnant patients. On this basis, neither is recommended.

Transplacental metastasis is exceedingly rare, but careful routine gross and histological evaluation of the placenta is recommended for every patient with a primary or metastatic melanoma diagnosed during pregnancy. ${ }^{11}$ Even if children born to mothers with advanced melanoma show no evidence of disease at birth, they should be closely monitored.

\section{Prognosis}

There is insufficient evidence to determine the prognostic significance of pregnancy on a melanoma diagnosis, and consensus is lacking because of

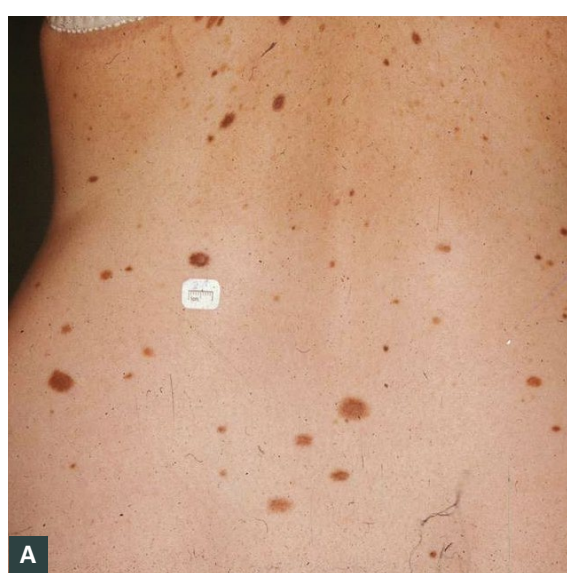

Figure 4. While they are potential precursors to melanoma, most dysplastic naevi will not evolve into melanoma. However, their presence defines a high-risk population for melanoma, and these women should be monitored closely during pregnancy.

A. A woman with dysplastic naevus syndrome; B. Dermoscopic appearance of a dysplastic naevus in a patient early in pregnancy; $\mathbf{C}$. An increased number and changed appearance of the peripheral dot pattern later in her pregnancy

Figure 4 courtesy of Dr Pascale Guitera

conflicting data. Available studies on the topic have been primarily retrospective cohort or case control studies, some of which did not control for known prognostic factors such as sun exposure or tumour thickness. Additionally, there are variable definitions of PAM, some including diagnoses made anywhere up to five years postpartum. As a consequence, it is difficult to truly assess the impact of pregnancy on melanoma-specific survival. Given the infrequent incidence of PAM and the need to provide care for both the mother and the baby, it is recommended that advice on the management of patients be obtained from specialist melanoma treatment centres.

\section{Special considerations}

As pregnancy is a period of change in the hormonal milieu and relative immunosuppression, there was initial concern that pregnancy might activate quiescent micrometastases from a previously treated melanoma. Current evidence does not support an adverse effect of pregnancy subsequent to successful treatment of melanoma
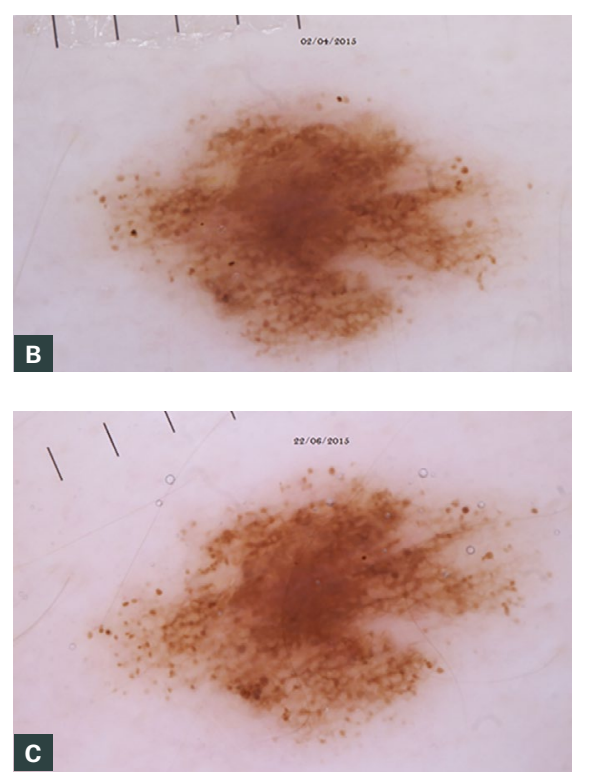

(c) The Royal Australian College of General Practitioners 2019 on either recurrence-free or overall survival, ${ }^{12}$ but high-quality data are lacking. Consensus guidelines developed by Cancer Council Australia suggest that 'while pregnancy does not appear to increase the risk of melanoma recurrence, women who are planning pregnancy following treatment of a high-risk melanoma should be made aware of their risk of recurrent melanoma, particularly within the first two to five years of diagnosis'. ${ }^{9}$

\section{Conclusions}

The assessment and management of pigmented skin lesions in pregnant women can be challenging. A sound knowledge of common pregnancy-associated changes and worrying features in pigmented lesions, which mirror those seen in the non-pregnant population, is essential for the GP. Treatment should parallel the treatment that would be offered to a non-pregnant woman, but with the avoidance of possible teratogens (blue dye for lymphatic mapping during sentinel node biopsy, and anaesthetic agents, especially during the first trimester) in an effort to optimise outcomes for both the mother and her baby.

\section{Key points}

- Pigmented skin lesions in pregnant women often present the GP with a clinical challenge.

- While a variety of physiological changes occur in this population, worrying features or significant changes in pigmented lesions should not be automatically attributed to pregnancy.

- The possibility of melanoma must always be considered when conventional ABCDE diagnostic criteria arousing suspicion or concern are present, because a delay in diagnosis can have serious adverse consequences.

- If a lesion is biopsied, it should be noted on the pathology form that the patient is currently pregnant or has recently been pregnant. 


\section{Authors}

Erica B Friedman MD, MS, Assistant Professor of Surgery, Division of Surgical Oncology, NYU Langone Health, New York, NY, USA

Richard A Scolyer MD, Co-Medical Director, Melanoma Institute Australia, University of Sydney, Sydney, NSW; Clinical Professor, Discipline of Pathology, Faculty of Medicine and Health, University of Sydney, Sydney, NSW; Senior Staff Specialist in Anatomical Pathology, Tissue Pathology and Diagnostic Oncology, Royal Prince Alfred Hospital, Sydney, NSW

John F Thompson MD, Chairman, Australian Melanoma Clinical Practice Guidelines Working Party; Professor of Melanoma and Surgical Oncology, University of Sydney, Sydney, NSW; Surgeon, Royal Prince Alfred Hospital and Melanoma Institute Australia, Sydney, NSW. john.thompson@melanoma.org.au

Competing interests: RS reports membership on the advisory board for Merck Sharp \& Dohme, Novartis,

Myriad and NeraCare, outside the submitted work. JT reports membership on the advisory board for and honoraria from GlaxoSmithKline, BMS, Provectus and MSD, as well as travel support from GlaxoSmithKline and Provectus, outside the submitted work.

Funding: None.

Provenance and peer review: Commissioned, externally peer reviewed.

\section{Acknowledgments}

The authors thank Kaye Oakley for her assistance in manuscript preparation.

\section{References}

1. Zampino MR, Corazza M, Costantino D, Mollica G, Virgili A. Are melanocytic nevi influenced by pregnancy? A dermoscopic evaluation. Dermatol Surg 2006;32(12):1497-504. doi: 10.1111/j.15244725.2006.32362.x

2. Aktürk AS, Bilen N, Bayramgurler D, Demirsoy EO, Erdoğan S, Kiran R. Dermoscopy is a suitable method for the observation of the pregnancyrelated changes in melanocytic nevi. J Eur Acad Dermatol Venereol 2007;21(8):1086-90. doi: 10.1111/j.1468-3083.2007.02204.x.

3. Sanchez JL, Figueroa LD, Rodriguez E. Behavior of melanocytic nevi during pregnancy. Am J Dermatopathol 1984;6(Suppl 6):89-91.

4. Foucar E, Bentley TJ, Laube DW, Rosai J. A histopathologic evaluation of nevocellular nevi in pregnancy. Arch Dermatol 1985;121(3):350-54.

5. Ellis DL. Pregnancy and sex steroid hormone effects on nevi of patients with the dysplastic nevus syndrome. J Am Acad Dermatol 1991;25(3):467-82. doi: 10.1016/01909622(91)70227-S.

6. Lee $Y Y$, Roberts $C L$, Dobbins $T$, et al. Incidence and outcomes of pregnancy-associated cancer in Australia, 1994-2008: A population-based linkage study. BJOG 2012;119(13):1572-82. doi: 0.1111/j.1471-0528.2012.03475.x.

7. Bannister-Tyrrell M, Roberts $C L$, Hasovits $C$, Nippita T, Ford JB. Incidence and outcomes of pregnancy-associated melanoma in New South Wales 1994-2008. Aust N Z J Obstet Gynaecol 2015;55(2):116-22. doi: 10.1111/ajo.12279.

8. Travers RL, Sober AJ, Berwick M, Mihm MC Jr, Harnhill RL, Duncan LM. Increased thickness of pregnancy-associated melanoma. Br J Dermatol 1995;132(6):876-83. doi: 10.1111/j.1365-2133.1995. tb16942.x.
9. Cancer Council Australia. Clinical practice guidelines for the diagnosis and management of melanoma. Sydney: Cancer Council Australia,2019. Available at https://wiki.cancer. org.au/australia/Guidelines:Melanoma [Accessed 12 June 2019].

10. Keleher A, Wendt R III, Delpassand E, Stachowiak AM, Kuerer HM. The safety of lymphatic mapping in pregnant breast cancer patients using Tc-99m sulfur colloid. Breast J 2004;10(6):492-95. doi: 10.1111/ j.1075-122X.2004.21503.x.

11. Balch CM, Houghton AN, Sober AJ, Soong S-J, Atkins MB, Thompson JF. Cutaneous melanoma. 5th edn. Saint Louis, MO: CRC Press, 2009.

12. Byrom L, Olsen CM, Knight L, Khosrotehrani K, Green AC. Does pregnancy after a diagnosis of melanoma affect prognosis? Systematic review and meta-analysis. Dermatol Surg 2015;41(8):875-82. doi: 10.1097/ DSS.0000000000000406. 